\title{
DIRETRIZES ESTRATÉGICAS BASEADAS NOS FATORES CRÍTICOS DE SUCESSO DA GESTÃO DO CONHECIMENTO VOLTADAS ÀS CARACTERÍSTICAS DA MEMÓRIA ORGANIZACIONAL
}

\section{STRATEGIC GUIDELINES BASED ON THE CRITICAL SUCCESS FACTORS OF KNOWLEDGE MANAGEMENT FOCUSED ON THE CHARACTERISTICS OF ORGANIZATIONAL MEMORY}

leda Pelógia Martins Damian ${ }^{1}$

María Manuela Moro Cabero²

\begin{abstract}
RESUMO
Apesar do conhecimento ser reconhecido como recurso estratégico em um contexto repleto de mudanças e desafios, as organizações ainda encontram muitas dificuldades para administrá-lo. Por vezes, a implantação da gestão do conhecimento não é bem sucedida porque seus fatores críticos de sucesso não são analisados de modo adequado. Além disso, estes fatores não consideram a memória organizacional, um conceito diretamente relacionado com a gestão do conhecimento. Com a intenção de contribuir para mais casos de sucesso relacionados a implantação da gestão do conhecimento nas organizações, este estudo tem por objetivo apresentar diretrizes estratégicas baseadas nos fatores críticos de sucesso da gestão do conhecimento que considerem as características da memória organizacional. Para tanto, além de um levantamento bibliográfico, foram realizadas entrevistas com gestores de organizações espanholas, com 0 intuito de compreender 0 contexto base para o desenvolvimento destas diretrizes. Após a realização das entrevistas, se conclui que as diretrizes estratégicas propostas são fundamentais para o sucesso da implantação da gestão do conhecimento.
\end{abstract}

Palavras-chave: Gestão do Conhecimento. Memória Organizacional. Fatores Críticos de Sucesso. Diretrizes. Estratégia.

\section{ABSTRACT}

Although knowledge is recognized as a strategic resource in a context full of challenges, organizations still find it very difficult to manage it. Sometimes, the implementation of knowledge management is not successful because its critical success factors are not adequately analyzed. In addition, these factors don't consider organizational memory, a concept directly related to knowledge management. With the intention of contributing to more successful cases related to the implementation of knowledge management in organizations, this study aims to present strategic guidelines based on the critical success factors of knowledge management that consider the characteristics of organizational memory. To this end, in addition to a bibliographic survey, interviews were conducted with managers of Spanish organizations, in order to understand the basic context for the development of these guidelines. After conducting the interviews, it is concluded that the proposed strategic guidelines are fundamental for the successful implementation of knowledge management. Keywords: Knowledge management. Organizational Memory. Critical Success Factors. Guidelines. Strategy.

1 Professora permanente no Programa de Pós Graduação em Ciência da Informaç̧ão. Universidade Estadual Paulista Júlio de Mesquita Filho, Brasil. ORCID https://orcid.org/0000-0001-5364-3243. E-mail: iedapm@usp.br

2 Professora Titular no Departamento de Biblioteconomia e Documentação. Universidade de Salamanca, Espanha. ORCID https:// orcid.org/0000-0001-5301-1924. E-mail: moroca@usal.es 


\section{INTRODUÇÃo}

Apesar das organizações já terem se conscientizado do papel estratégico do conhecimento para se manterem e se destacarem no ambiente em que atuam, a implantação de sua gestão ainda representa um grande desafio. Muitas organizações falham ao implantarem a Gestão do Conhecimento (GC) porque não se atentam aos fatores críticos de sucesso associado a esta gestão, bem como a memória organizacional, conceito indissociável a GC.

0 conhecimento, para Souza, Dias e Nassif (2011), ganha espaço nas discussões e práticas organizacionais como recurso estratégico para as organizações se manterem no mercado e buscarem uma competitividade sustentável. Para os autores, a GC envolve o entendimento de como as pessoas, a informação e o conhecimento se relacionam dinamicamente. A GC, conforme Freire et al. (2012), se trata de uma ferramenta de suporte para a criação e para a manutenção do conhecimento organizacional, já que faz com que o conhecimento das pessoas seja compartilhado, registrado e preservado como M0 e, posteriormente, disseminado.

Castillo e Cazarini (2009) afirmam que a implantação da GC tem levado importantes benefícios para algumas organizações, mas fracassos para muitas outras que não conseguiram empregá-la de modo adequado. Para que mais casos de sucesso sejam relacionados a implantação da GC, é preciso considerar seus Fatores Críticos de Sucesso (FCS). 0 mapeamento e entendimento dos FCS, conforme Gnecco Jr. et al. (2010), são cruciais para a execução da GC, pois retratam áreas em que os resultados, se forem satisfatórios, garantem o desempenho competitivo de sucesso para a organização.

Além dos FCS da GC, é preciso considerar a importância da Memória Organizacional (M0), que pode ser vista na definição do seu conceito dada por Freire et al. (2012, p. 43). Para os autores, a M0

[...] trata-se da criação de um acervo com informações, sendo também uma espécie de "ferramenta" da organização para o gerenciamento do conhecimento e das tarefas individuais e do grupo. A memória organizacional leva ao compartilhamento e reutilização do conhecimento da empresa, do conhecimento individual e das habilidades do saber fazer as tarefas da organização.

A M0, segundo Pereira, Silva e Pinto (2016), diz respeito a representação explícita do conhecimento e de informações relevantes ao contexto organizacional, com 0 intuito de facilitar 0 acesso, o compartilhamento e a reutilização destes recursos entre os membros da organização.

Diante do exposto, se acredita que este estudo que objetiva desenvolver diretrizes estratégicas baseadas nos FCS da gestão do conhecimento que considerem as características da MO possa contribuir 
para o campo da Ciência da Informação (Cl), área que carece de estudos voltados aos inúmeros elementos e conceitos que interagem com a GC. Foram realizadas entrevistas com os gestores de organizações espanholas para que as diretrizes estratégicas baseadas nos FCS da GC que considerem as características da MO pudessem ser desenvolvidas.

As diretrizes estratégicas baseadas nos FCS da GC que considerem as características da MO representam uma oportunidade de desenvolver uma vantagem sustentável em um ambiente altamente competitivo, além de oferecer uma série de outros benefícios como redução de custos, eliminação de retrabalho, inovação, aumento de produtividade, melhoria da qualidade, etc.

A fim de alcançar os objetivos propostos, foi realizado um levantamento bibliográfico sobre os conceitos chave para a pesquisa que são: gestão do conhecimento, fatores críticos de sucesso da gestão do conhecimento e memória organizacional. Na metodologia da pesquisa foi utilizada a técnica qualitativa da análise de conteúdo para a análise dos dados coletados por meio das entrevistas realizadas junto aos gestores das organizações espanholas.

\section{REFERENCIAL TEÓRICO}

\subsection{Fatores críticos de sucesso da gestão do conhecimento}

0 conhecimento, de acordo com Bukowitz e Williams (2002), contribui para o desenvolvimento de impactos positivos nos resultados financeiros, para a inovação e para a geração de valor ao cliente. A GC envolve "um conjunto de processos que governa a aquisição, a criação, o compartilhamento, o armazenamento e a utilização de conhecimento no âmbito das organizações" (ANGELONI, 2008, p.2).

A GC, segundo Pérez-Montoro (2008), se refere a gestão de todos os ativos intelectuais envolvidos no contexto organizacional, considerando como ativo aquele que auxilia ou que permite cumprir com os objetivos da organização. Para Hoffmann (2016), a GC pode ser entendida como um processo organizacional ou um modelo de gestão focado na estratégia da organização com o diferencial competitivo de envolver abordagens como a gestão do capital intelectual, a aprendizagem organizacional, a gestão por competências, a gestão da inovação e a inteligência organizacional ou competitiva. 
A importância da GC para as organizações, conforme Earl (2001), se deve ao fato de ser uma das principais atividades envolvidas no processo de inovação de produtos e processos, para a tomada de decisão em nível estratégico e pela possibilidade de adaptação e renovação organizacional. Para que as organizações possam usufruir dos benefícios da GC, é importante que tenham consciência dos obstáculos que podem surgir durante sua implantação e comprometer 0 alcance dos objetivos esperados.

Os fatores, as atividades ou as práticas existentes ou a serem desenvolvidas, atributos ou características que podem influenciar o sucesso ou falha de uma iniciativa de implantação da GC devem, para Wong (2005), ser analisados e trabalhados como fatores críticos para 0 seu sucesso. Os FCS, para Furlanetto e Oliveira (2008), são o caminho mais seguro para as organizações atingirem melhores resultados e sustentabilidade em longo prazo, ou seja, representam áreas em que resultados satisfatórios asseguraram o desempenho organizacional.

Para Gnecco Jr. et al. (2010), o estudo, mapeamento e compreensão dos FCS são obrigatórios para a implantação da GC, e afirmam que, apesar de existir uma diversidade de FCS na literatura, se percebe uma convergência em torno de um conjunto de FCS fundamentais para implementação de estratégias de GC bem-sucedidas. Para Del-Massa, Damian e Valentim (2018), os FCS são aspectos relevantes da GC, devem ser continuamente monitorados e podem abranger desde a comunicação, tecnologia, pessoas, processos até aspectos de liderança.

Com base nos autores citados acerca dos FCS da GC, elaborou-se o Quadro um, onde se destacam os dez FCS mais citados, bem como seus autores. Os FCS são apresentados seguindo a ordem do mais citado para o menos citado de acordo com a pesquisa realizada. 
Quadro 1 - Fatores Críticos de Sucesso da Gestão do Conhecimento

\begin{tabular}{|c|c|}
\hline $\begin{array}{l}\text { Fator crítico de } \\
\text { sucesso }\end{array}$ & Autores \\
\hline Pessoas & $\begin{array}{l}\text { Davenport e Prusak (1998); Nonaka e Takeuchi (1997); Davenport e Prusak } \\
\text { (1998); Wong e Aspinwall (2004); Chong e Choi (2005); Dalkir (2005); Hung et } \\
\text { al. (2005); Wong (2005); Terra (2005); Furlanetto e Oliveira (2008); Castillo e } \\
\text { Cazarini (2009); Fresneda et al. (2009); Garcia e Possamai (2009); Gnecco Jr. et } \\
\text { al. (2010); Batista (2012); Besen (2013); Rivera e Rivera (2016); Correa, Ziviani e } \\
\text { Martins (2018) }\end{array}$ \\
\hline Cultura & $\begin{array}{l}\text { Davenport e Prusak (1998); Wong e Aspinwall (2004); Chong e Choi (2005); Hung } \\
\text { et al. (2005); Terra (2005); Wong (2005); Furlanetto e Oliveira (2008); Castillo e } \\
\text { Cazarini (2009); Fresneda et al. (2009); Garcia e Possamai (2009); Gnecco Jr. et } \\
\text { al. (2010); Besen (2013); Rivera e Rivera (2016); Correa, Ziviani e Martins (2018) }\end{array}$ \\
\hline Tecnologia & $\begin{array}{l}\text { Davenport e Prusak (1998); Wong e Aspinwall (2004); Chong e Choi (2005); } \\
\text { Dalkir (2005); Hung et al. (2005); Terra (2005); Furlanetto e Oliveira (2008); } \\
\text { Castillo e Cazarini (2009); Gnecco Jr. et al. (2010); Batista (2012); Besen (2013); } \\
\text { Rivera e Rivera (2016); Correa, Ziviani e Martins (2018) }\end{array}$ \\
\hline $\begin{array}{l}\text { Mensuração } \\
\text { dos Resultados }\end{array}$ & $\begin{array}{l}\text { Davenport e Prusak (1998); Wong e Aspinwall (2004); Chong e Choi (2005); Terra } \\
\text { (2005); Wong (2005); Furlanetto e Oliveira (2008); Garcia e Possamai (2009); } \\
\text { Gnecco Jr. et al. (2010); Besen (2013); Rivera e Rivera (2016); Correa, Ziviani e } \\
\text { Martins (2018) }\end{array}$ \\
\hline Liderança & $\begin{array}{l}\text { Davenport e Prusak (1998); Wong e Aspinwall (2004); Chong e Choi (2005); } \\
\text { Dalkir (2005); Hung et al. (2005); Castillo e Cazarini (2009); Gnecco Jr. et al. } \\
\text { (2010); Batista (2012); Besen (2013); Rivera e Rivera (2016); Correa, Ziviani e } \\
\text { Martins (2018) }\end{array}$ \\
\hline Processos & $\begin{array}{l}\text { Davenport e Prusak (1998); Wong e Aspinwall (2004); Dalkir (2005); Wong } \\
\text { (2005); Furlanetto e Oliveira (2008); Castillo e Cazarini (2009); Garcia e Possamai } \\
\text { (2009); Gnecco Jr. et al. (2010); Batista (2012); Besen (2013); Correa, Ziviani e } \\
\text { Martins (2018) }\end{array}$ \\
\hline $\begin{array}{l}\text { Alta } \\
\text { administração }\end{array}$ & $\begin{array}{l}\text { Davenport e Prusak (1998); Chong e Choi (2005); Hung et al. (2005); Wong } \\
\text { (2005); Terra (2005); Furlanetto e Oliveira (2008); Castillo e Cazarini (2009); } \\
\text { Garcia e Possamai (2009); Gnecco Jr. et al. (2010); Correa, Ziviani e Martins } \\
\text { (2018) }\end{array}$ \\
\hline Estratégia & $\begin{array}{l}\text { Davenport e Prusak (1998); Wong e Aspinwall (2004); Wong (2005); Castillo e } \\
\text { Cazarini (2009); Fresneda et al. (2009); Garcia e Possamai (2009); Gnecco Jr. et } \\
\text { al. (2010); Besen (2013); Correa, Ziviani e Martins (2018) }\end{array}$ \\
\hline Infraestrutura & $\begin{array}{l}\text { Wong e Aspinwall (2004); Terra (2005); Furlanetto e Oliveira (2008); Castillo e } \\
\text { Cazarini (2009); Garcia e Possamai (2009); Gnecco Jr. et al. (2010); Besen (2013); } \\
\text { Rivera e Rivera (2016) }\end{array}$ \\
\hline Recursos & $\begin{array}{l}\text { Davenport e Prusak (1998); Holsapple e Joshi (1999); Garcia e Possamai (2009); } \\
\text { Besen (2013); Correa, Ziviani e Martins (2018) }\end{array}$ \\
\hline
\end{tabular}

Fonte: elaborado pelas autoras. 
As pessoas são consideradas um importante viabilizador da GC porque são elas que captam, criam, armazenam, compartilham e aplicam conhecimento. Nonaka e Takeuchi (1997) ressaltam que as organizações não podem criar conhecimento senão pelas pessoas, elas são as únicas capazes de realizar tal feito. As organizações precisam reconhecer o papel fundamental que as pessoas desempenham nos processos de GC e criar condições para que elas possam contribuir para a criação do conhecimento organizacional. Diante disso, os programas de educação e capacitação e o desenvolvimento de carreiras são essenciais para aumentar a capacidade dos colaboradores na execução dos processos de GC e, assim, contribuírem para a melhoria do desempenho organizacional (BATISTA, 2012).

A cultura organizacional, conforme Furlanetto e Oliveira (2008), pode direcionar as ações operacionais e a maior ou menor agregação do conhecimento. Para Davenporte Prusak (1998), a cultura organizacional é dependente dos sujeitos organizacionais. Wong (2005) afirma que a implantação da GC deve estar alinhada à cultura organizacional vigente, ou esta deverá ser mudada de forma a viabilizar a implantação de tal processo.

A tecnologia é essencial para a implantação da GC, pois permite agilizar os processos de GC por meio de ferramentas desenhadas para criar, armazenar, compartilhar e aplicar conhecimento (BATISTA, 2012). Furlanetto e Oliveira (2008) afirmam que tecnologia representa toda a estrutura tecnológica, 0 que envolve softwares e bancos de dados que registram e permitem a disseminação de conhecimento.

A mensuração dos resultados, de acordo com Furlanetto e Oliveira (2008), possibilita, a partir da definição de métricas, avaliar os investimentos em GC e identificar a aderência da GC aos objetivos de negócio, além de ser um estímulo à manutenção e ao incremento dos valores aplicados. Para Wong (2005), a avaliação de benefícios e a criação de valor relacionados a GC possibilita aos membros da organização perceber os benefícios por ela gerados.

A liderança, para Batista (2012), desempenha papel estratégico na implantação da GC porque apresenta e reforça a visão, os objetivos e as estratégias de GC, além de ser exemplo nas áreas de compartilhamento do conhecimento e de trabalho colaborativo.

A GC precisa ser incorporada à gestão de processos, porque, conforme Batista (2012), processos sistemáticos e modelados com o conhecimento adequado contribuem para melhorar o desempenho organizacional. Para Wong (2005), a gestão dos processos da GC diz respeito a essência do que pode ser feito com o conhecimento nas organizações. A sistematização dos processos, de acordo com Furlanetto e Oliveira (2008, p. 107), "é uma prática direcionada à qualidade dos produtos e serviços e à busca de satisfação dos clientes". 
A alta administração, segundo Furlanetto e Oliveira (2008), é responsável por mobilizar os demais níveis hierárquicos, por meio do suporte ao compartilhamento do conhecimento, da criação de condições internas para a disseminação do conhecimento, da definição clara de metas, objetivos e resultados esperados, e da liberdade de interação entre as pessoas e grupos. Wong (2005) reforça que o suporte da alta direção é essencial para GC, já que sua implantação requer uma profunda alteração organizacional.

A estratégia disponibiliza a organização o fundamento de como suas capacidades e recursos devem ser desenvolvidos para que os objetivos da GC sejam alcançados (WONG, 2005). As estruturas organizacionais são um processo contínuo de adequação ao contexto de mercado e envolvem aspectos como mudanças internas, descentralização de tarefas, autonomia e delegação de poder na motivação para 0 aprendizado, redução de níveis hierárquicos e desempenho vinculado à satisfação dos clientes (FURLANETTO; OLIVEIRA, 2008).

Os recursos financeiros são necessários para a implantação da GC. Para a disponibilização dos recursos financeiros, segundo Correa, Ziviani e Martins (2018), devem ser considerados aspectos como infraestrutura, sistemas tecnológicos, salários e insumos materiais. Ainda de acordo com os autores, é preciso disponibilizar tempo para que os colaboradores possam compartilhar conhecimento e para que a equipe de GC realize suas atividades.

Holsapple e Joshi (1999) propuseram três classes de influências para o sucesso da GC nas organizações que são: 1) Gestão, constituída por coordenação, controle, medição e liderança; 2) Recursos, que diz respeito ao conhecimento e demais recursos (humanos, materiais e financeiros); e 3) Meio ambiente, que engloba os fatores vinculados à concorrência, aos mercados, à pressão do tempo, aos aspectos governamentais e climas econômicos, etc.

Os FCS da GC destacados por Wong e Aspinwall (2004) são: apoio da liderança; cultura favorável; tecnologia da informação; estratégia e propósitos; avaliação e medição; infraestrutura organizacional; processos e atividades; fatores motivacionais; recursos e pesquisas; educação e treinamento; e gestão de recursos humanos. Para Chong e Choi (2005), os FCS da GC são: treinamento e envolvimento dos colaboradores; trabalho em equipe; empoderamento dos colaboradores; liderança e comprometimento da alta administração; infraestrutura de sistemas de informação; mensuração de desempenho; cultura favorável; benchmarking; estrutura de conhecimento; e eliminação das limitações organizacionais.

Liderança, pessoas, processos e tecnologias são, para Dalkir (2005), os principais FCS da GC. Hung et al. (2005) destacaram sete FCS da GC: estratégia de benchmarking e estrutura de conhecimento; 
cultura organizacional; tecnologia da informação; envolvimento dos trabalhadores e formação; liderança e compromisso da alta administração; ambiente de aprendizagem e controle de recursos; e avaliação da formação profissional e trabalho em equipe.

Para Terra (2005), os FCS da GC são: alta administração; políticas de recursos humanos; cultura organizacional; estrutura organizacional; sistema de informação; e mensuração de resultados. Castillo e Cazarini (2009) destacam os seguintes FCS da GC: estratégia de GC alinhada à estratégia da organização; cultura organizacional de conhecimento; sistemas de informação; envolvimento e treinamento dos funcionários; liderança e compromisso de gerentes de alto nível; estabelecimento de atividades, funções e processos e avaliação do desempenho; e benchmarking das práticas de GC.

Os seguintes FCS da GC foram destacados por Garcia e Possamai (2009): plataforma de trabalho; suporte da alta direção; necessidades e oportunidades da GC; alinhamento com objetivos estratégicos; infraestrutura definida para GC; processos definidos para GC; investimento em recursos; avaliação e medição de resultados; cultura organizacional; capacitação para GC; e políticas motivacionais.

Gnecco Jr. et al. (2010) criaram uma lista com 20 FCS da GC: 1) Liderança e comprometimento da alta administração; 2) GC com base na medição de desempenho; 3) Tecnologia, infraestrutura de sistemas de informação, sistema de gestão de infraestrutura de GC; 4) Fatores motivacionais, compartilhamento de conhecimento, envolvimento dos trabalhadores, incentivos e recompensas; 5) Cultura amigável de conhecimento; 6) Políticas, processos, atividades e procedimentos, reengenharia de processos, processo de negócio e proposição de valor; 7) Programas de Treinamento; 8) Estratégia, estratégia do conhecimento, GC como uma iniciativa estratégica; 9) Redes de peritos, equipe específica, apoio dos administradores experientes; 10) Definição de critérios para o conteúdo selecionado; 11) Arquitetura do Conhecimento, estrutura de conhecimento; 12) Empowerment; 13) Infraestrutura organizacional; 14) Eliminação das barreiras organizacionais; 15) Alinhamento entre estratégia de GC e estratégia dos negócios; 16) Abordagem holística/sistêmica da gestão do conhecimento; 17) Imitar as empresas líderes em GC; 18) Gestão da mudança e impacto da comunicação; 19) GC em todo 0 seu ciclo de vida; e 20) Recursos e Pesquisas.

Para Besen (2013), os seguintes FCS devem serconsiderados: apoio e liderança gerencial; cultura; tecnologia da informação; estratégia e objetivo; medição; infraestrutura organizacional; processos e atividades; auxílio motivacional; recursos; treinamento e educação; e gestão de RH. Rivera e Rivera (2016) identificaram seis facilitadores ou FCS da GC: liderança, cultura, estrutura, recursos humanos, tecnologias da informação e comunicação, e medição. Correa, Ziviani e Martins (2018) destacam de 
13 FCS voltados a implantação da GC: estratégia; liderança e suporte da alta administração; equipe de GC; recursos (financeiro, humano, material, tempo); processos e atividades; gestão de recursos humanos; treinamento e educação; motivação; trabalho em equipe; cultura; tecnologia da informação; mensuração e projeto piloto.

Apesar de muitos estudos se dedicarem aos FCS da GC, se verifica que não existem referências à memória organizacional, conceito estritamente ligado à GC. Com o objetivo de desenvolver diretrizes estratégicas que minimizem tal lacuna, se discorre, a seguir, sobre o importante papel da MO na GC.

\subsection{0 papel da memória organizacional}

Para que possam desenvolver vantagens competitivas, Neves e Cerdeira (2018) afirmam que as organizações precisam criar, utilizar e preservar a memória dos conhecimentos acumulados em seu histórico e em seus processos organizacionais. Para os autores, a preservação desta memória permite compartilhar e manter 0 conhecimento da organização, independentemente da rotatividade dos seus membros. A MO, para Spiller e Pontes (2007), é um conjunto de conhecimentos derivados das experiências das pessoas, da cultura, dos processos e dos documentos. Deste modo, a MO é influenciada, entre outras coisas, por pessoas, cultura e processos, assim como ocorre com os FCS da GC.

A memória organizacional

pode ser entendida como a habilidade das organizações para salvar, reter e fazer uso de informações do passado nas atividades atuais. É um elemento chave que permite que as organizações aprendam dos erros e acertos do passado. Mas fazer uso de este tipo de informação é uma atividade complexa, pois se encontra dispersa dentro da organização em diversos lugares (i.e., nos indivíduos, em documentos, em relatórios, sistemas de informação, etc.) e é heterogênea (i.e., conhecimentos tácito, explícito, mentalizado, codificado, incorporado, embutido, declarativo, procedural, condicional, etc.) (MELGAR-SASIETA; BEPLER; PACHECO, 2011, p. 1).

Para que as organizações possam usufruir dos benefícios da M0, Neves e Cerdeira (2018) destacam a necessidade de identificar e localizar os conhecimentos relevantes em seus ambientes, uma vez que, sem tais ações, a criação de mecanismos de obtenção e armazenamento de conhecimento se torna inviável.

Os FCS enumerados para gerir o conhecimento, são igualmente reconhecíveis para a M0. Assim, é preciso conhecer os contextos organizacionais para identificar as necessidades operacionais, legais e de negócio; dispor de uma direção estratégica comprometida e reconhecida pela alta 
direção na organização. Neste sentido, missão, objetivos e políticas para criar, capturar, gerir, aceder e preservar a MO devem ser formulados, implementados e atualizados; tanto em nível operacional como organizacional. Além disso, a MO deve administrar os recursos disponíveis para a sua gestão e preservação, o que inclui controle dos processos, coordenação dos recursos, implementação das estratégias e medição de resultados, assim como sua melhoria continua. Finalmente, cabe dizer que a gestão da MO deve dispor de modelos padronizados certificáveis para avaliar a sua maturidade (como a ISO 30301), qualidade (como a ISO 9001), etc.

A MO se cria e se preserva sobre uma estrutura tecnológica submetida a elevada obsolescência, a qual exige novos requisitos e atuações favorecedoras de uma arquitetura de processos adaptáveis as mudanças.

Se faz oportuno destacar como a GC influencia e é influenciada pela M0, o que justifica considerar as características da MO na proposição de diretrizes estratégicas baseadas nos FCS da GC. A GC, para Freire et al. (2012), propicia o compartilhamento, a explicitação e armazenamento do conhecimento e, sua criação e manutenção, dependem da M0.

Para Nascimento e Vitoriano (2017), a MO contribui para o sucesso da implantação da GC e propicia o registro e a disseminação do conhecimento existente nas organizações. Para as autoras, uma das funções da MO é dar suporte aos processos de GC, uma vez que a MO está ligada à GC por meio da transformação de seus repositórios em repertórios de conhecimento, que contribuem para 0 processo decisório e possibilitam a obtenção de vantagem competitiva. A MO é, conforme Nascimento et al. (2016), uma ferramenta para a GC, pois leva ao compartilhamento e reutilização do conhecimento organizacional, e a GC possibilita a constituição da MO para uso e reuso.

Santos, Moro-Cabero e Valentim (2016) afirmam que existe um importante diálogo entre a MO e a GC e destacam que, a partir do potencial estratégico da MO e do conhecimento, é possível desenvolver aprendizagens essenciais e disponibilizar diferencial estratégico para o processo decisório. Freire et al. (2012) afirmam que, por meio da M0, a organização preserva o conhecimento de seus membros, o que Ihe permite repetir experiências bem-sucedidas e evitar erros anteriormente cometidos.

Arboníes (2006) ressalta que uma das maneiras que as organizações têm para transferir as competências de seus colaboradores para os níveis organizacionais é por meio da M0. Para Arboníes e Aldázabal (2005), a GC envolve a integração de fatores geradores de valor agregado, dentre os quais se destaca a MO, demonstrando, assim, a importância e a estreita relação entre os conceitos desenvolvidos nesta pesquisa. 
A sua vez, a MO preserva o conhecimento para o desenvolvimento dos processos de negócio e favorece o processamento dos dados, dos registros e das informações derivados desses processos, facilitando sua transparência, a prestação de contas, a evidência dos mesmos, tornandose fundamental para atingir os resultados desejados. A MO é sustento da governança organizacional, apoia a infraestrutura do conhecimento, a transparência, a gestão de riscos, a prestação de contas, limita a corrupção, assegura a informação, apoia os processos avaliadores e certificadores; em suma, os princípios democráticos organizacionais.

Para Benavides e Quintana (2003), as organizações, ao reconhecerem a importância do conhecimento, passam por uma mudança substancial em suas gestões, o que inclui, entre outras coisas, cuidar do armazenamento do conhecimento organizacional que se dá por meio da constituição e manutenção de sua memória organizacional.

\section{PROCEDIMENTOS METODOLÓGICOS}

A pesquisa é de natureza qualitativa, caracterizada como descritiva e exploratória, cujo universo de pesquisa são as empresas do setor cárnico de Salamanca, Espanha. Apresenta um delineamento desenvolvido em 3 fases: constituição do corpus teórico-metodológico; construção do corpus empírico; e análise e interpretação dos resultados.

$\mathrm{Na}$ primeira fase, foram tomadas como referência as principais obras e autores da $\mathrm{Cl}$ que dizem respeito a GC, MO e FCS da GC, o que permitiu levantar os contextos da MO e da GC a serem considerados pelas diretrizes estratégicas baseadas nos FCS da GC. A segunda fase se dedicou a coleta de dados relacionados aos FCS identificados na fase anterior. Uma pesquisa exploratória foi realizada, onde se buscou informações sobre o contexto organizacional acerca dos FCS da GC que tornasse possível realizar a proposição de diretrizes estratégicas baseadas nos FCS da GC que considerem as características da MO. Foram realizadas entrevistas semiestruturadas com gestores de empresas de Salamanca, na Espanha.

Foi realizada uma pesquisa qualitativa por meio da análise de conteúdo de Bardin (2011), com utilização da análise categorial, com categorias definidas à priori, compostas pelos principais FCS da GC identificados na revisão de literatura. 0 universo de pesquisa foi constituído por empresas do setor carníco de Salamanca, na Espanha. Esta escolha se baseou na relevância de tal setor, que, de acordo com a Asociación Nacional de Industrias de la Carne de España (ANICE), representa o quarto 
setor industrial de maior representatividade da Espanha, cuja produção ocupa o primeiro lugar da indústria espanhola de alimentos e bebidas (ANICE, 2019). Na Comunidad de Castilla y León, onde se localiza Salamanca, o setor cárnico, de acordo com a Junta de Castilla y León (JCyL), é referência, ocupa a primeira posição de produção e consumo e representa $26 \%$ da indústria agroalimentar, sendo determinante para a economia local (JCyL, 2019). Foram entrevistados nove gestores de distintas empresas do setor cárnico de Salamanca, Espanha.

A terceira fase se refere à análise e interpretação dos resultados obtidos, momento em que são retomados os objetivos e confrontados com o referencial teórico-metodológico que fundamenta 0 estudo, assim como as atividades aplicadas e exploratórias, com vistas à elaboração das conclusões a que a pesquisa chegou com relação às formulações iniciais.

Como procedimento de coleta de dados foi desenvolvido um roteiro para a realização da entrevista semiestruturada, composto por questões iniciais sobre a empresa e três questões sobre cada um dos FCS da GC identificados como os mais citados de acordo com a literatura pesquisada. Para a seleção das organizações participantes desta pesquisa, se procurou a Cámara Oficial de Comercio, Industria y Servicios de Salamanca que se dispôs a entrar em contato com gestores que poderiam colaborar com esta pesquisa.

\section{ANÁLISE E APRESENTAÇÃO DOS RESULTADOS}

Para análise dos dados coletados se utilizou a análise de conteúdo que, de acordo com Bardin (2011), diz respeito as iniciativas de explicitação, sistematização e expressão do conteúdo de mensagens, com o objetivo de obter deduções lógicas e justificadas a respeito dessas mensagens. Se fez uso da análise categorial com as categorias definidas ‘a priori’ que, para Bardin (2011), permite ao pesquisador ir a fase da coleta de dados destinado ao que necessita analisar, o que se adequa ao objetivo desta pesquisa voltada aos FCS da GC.

As empresas que participaram desta pesquisa se encontravam no mercado a mais de 15 anos, o que demonstra serem empresas consolidadas em um mercado estabelecido; apenas duas possuíam filiais e três estavam realizando análises para a abertura futura de filiais; todas tinham entre 6 a 49 funcionários; e possuíam natureza familiar.

De modo geral, os colaboradores dispunham dos conhecimentos de que as empresas necessitavam. Ainda que não dispusessem de políticas formais para motivar seus colaboradores a 
compartilharem conhecimentos, as empresas reconheciam a importância das pessoas como fontes geradoras de conhecimento e procuravam motivar o compartilhamento de conhecimento por meio do trabalho em equipe e de um ambiente que proporcionasse a participação de todos.

Todos os gestores afirmaram que a cultura das empresas estava alinhada com o processo de aprendizagem e com os programas de incentivos ao compartilhamento do conhecimento, que existia um clima organizacional positivo e que os colaboradores estavam comprometidos com a empresa.

Quando questionados sobre quais tecnologias dispunham para criar, armazenar, compartilhar e utilizar o conhecimento, os gestores citaram, basicamente, os sistemas de informação e a internet. Em relação a frequência com que essas tecnologias eram atualizadas e os colaboradores treinados para utilizá-las, se verificou que as atualizações se limitavam as atualizações dos sistemas de informação e 0 treinamento dos colaboradores para utilizar estes sistemas ocorriam basicamente na contratação de novos colaboradores ou quando ocorriam alterações substanciais no sistemas de informação que utilizavam.

Apesar de não existirem métricas definidas para os investimentos em GC, os gestores reconheciam a importância destas métricas como um estímulo à manutenção e ao incremento dos valores aplicados.

Como as empresas pesquisadas não dispunham da GC formalmente implantada, não foi possível verificar ações que permitissem afirmar que a alta administração mobilizava os demais níveis hierárquicos para que os processos de GC ocorressem de forma efetiva, ainda que, em alguns casos, foi relatado que a alta administração incentivava a realização de atividades que estavam diretamente ligadas a GC como 0 desenvolvimento do trabalho em equipe e o compartilhamento do conhecimento. Quando questionados sobre a liberdade que a alta administração dava para que ocorresse a interação entre as pessoas e grupos, foi possível identificar o caráter familiar e as características de empresas tradicionais e fortemente hierarquizadas se faziam presentes.

Uma característica que chamou a atenção durante as entrevistas foi o limitado papel influenciador desempenhado pelas lideranças. Para os gestores, tal fato se deve ao tamanho das empresas e ao seu caráter familiar. Apesar deste contexto, os gestores afirmaram que os líderes influenciavam ou poderiam influenciar os demais colaboradores a apresentarem um comportamento desejado para GC. Não foram encontrados processos formais de GC em nenhuma das empresas pesquisadas, ainda que o compartilhamento do conhecimento fosse fortemente incentivado. 
Todos os gestores entrevistados afirmaram que as empresas possuíam objetivos estratégicos claramente definidos, ainda que, na maioria dos casos, fosse possível verificar que tais objetivos fossem genéricos. Nenhuma empresa pesquisada tinha objetivos estratégicos da GC claramente definidos. Apesar de muito receptivos à GC, os gestores afirmaram que as empresas não dispunham de recursos (financeiro, material e tempo) para esta gestão. Dentre as barreiras citadas estavam a falta de recursos financeiros e de colaboradores.

A partir da análise dos dados coletados sobre os fatores críticos de sucesso da GC foi possível desenvolver as diretrizes estratégicas demonstradas a seguir.

\subsection{Diretrizes estratégicas baseadas nos fatores críticos de sucesso da gestão do conhecimento}

Tomando por base o levantamento bibliográfico realizado e os dados coletados por meio de entrevistas realizadas com gestores de empresas do setor cárnico de Salamanca, uma série de diretrizes estratégicas baseadas nos FCS da GC foram desenvolvidas. As diretrizes estratégicas são apresentadas de acordo com o FCS da GC em que foram baseadas.

\section{Pessoas}

- Desenvolver políticas para apoiar e estimular atividades que desencadeiam a criação de conhecimento individual e organizacional;

- $\quad$ Propiciar condições adequadas para que a conversão do conhecimento se dê de forma efetiva;

- Propor programas de capacitação profissional para 0 desenvolvimento de competências e habilidades voltadas aos objetivos organizacionais;

- Desenvolver políticas voltadas ao reconhecimento e a valorização dos talentos humanos;

- Criar incentivos e desenvolver políticas motivacionais e de recompensa que levem ao trabalho coletivo e ao compartilhamento de conhecimento;

- $\quad$ Formar equipes colaborativas ou redes de especialistas para de reunir o conhecimento;

- Criar condições para que indivíduos aprendam com suas experiências e, a partir daí, desenvolver a memória organizacional;

- Motivar e incentivar os indivíduos a compartilhar o que aprenderam em uma MO;

- $\quad$ Capacitar os indivíduos para atuarem em projetos de gestão do conhecimento; 
- Desenvolver uma gestão de recursos humanos focada na definição de políticas voltadas à aquisição, geração, difusão e armazenamento do conhecimento da organização;

- Disseminar informações sobre benefícios, política, estratégia, modelo, plano e ferramentas de gestão do conhecimento na organização para assegurar a participação dos indivíduos;

- Realizar processos de contratação de pessoas alinhados com os objetivos organizacionais;

- $\quad$ Fazer com que os indivíduos reconheçam o conhecimento como 0 ativo organizacional mais importante;

- Desenvolver atividades que permitam capturar o conhecimento não documentado, para, assim, incorpora-lo à memória organizacional;

- Instituir o planejamento formal para treinamentos e programas de aprendizado a serem ofertados aos colaboradores.

\section{Cultura}

- Fomentar uma cultura organizacional que atue como fator facilitador dos processos de criação, aquisição, armazenamento, compartilhamento e uso de conhecimento;

- Criar espaços criativos e favoráveis à troca de experiências e ao intercâmbio entre os indivíduos;

- Disseminar a cultura da visão compartilhada;

- Incentivar a criatividade;

- Desenvolver um sistema eficiente de comunicação organizacional;

- Desenvolver uma cultura organizacional que não seja resistente a mudanças;

- Alinhar a cultura organizacional ao processo de aprendizagem, aos programas de incentivos e a orientação positiva para o compartilhamento do conhecimento;

- $\quad$ Propiciar um clima organizacional positivo;

- C C Criar condições para que os indivíduos sejam comprometidos com a organização;

- Alinhar a implantação da gestão do conhecimento à cultura organizacional vigente ou alterar a cultura da organização de forma a viabilizar essa implantação;

- Criar uma cultura organizacional favorável ao desenvolvimento e a manutenção da MO, ou seja, uma cultura organizacional que valorize, incentive e premie o comprometimento dos indivíduos com a utilização e manutenção da memória organizacional. 


\section{Tecnologia}

- Dispor de tecnologias de informação e de comunicação que possam proporcionar a comunicação informacional de forma eficiente;

- Utilizar tecnologias de informação e comunicação para criar mecanismos de captação, sistematização e organização de conhecimento organizacional, de modo a estimular o seu uso e 0 seu compartilhamento;

- Dispor de tecnologia que possibilite acesso às informações em todos os níveis organizacionais;

- Investir em tecnologias voltadas à facilitação do aprendizado e da colaboração;

- Desenvolver um banco de conhecimentos da organização;

- Dispor de tecnologia adequada para o desenvolvimento e a manutenção da MO;

- $\quad$ Fazer uso das tecnologias de informação e comunicação para transformar o conhecimento individual em conhecimento organizacional;

- Dispor de tecnologias de informação e comunicação para que o compartilhamento do conhecimento possa ocorrer entre indivíduos alocados em espaços geográficos distintos;

- $\quad$ Fazer uso de sistemas de informação e de comunidades de prática para que o conhecimento possa ser compartilhado e disseminado;

- Dispor de sistemas de informação que direcionem a integração entre os sistemas para que os processos de geração, difusão e armazenamento de conhecimento sejam otimizados;

- Dispor de tecnologia que possa acelerar os processos de gestão do conhecimento por meio de ferramentas desenhadas para criar, armazenar, compartilhar e aplicar conhecimento;

- Fazer uso de tecnologias que auxiliam os processos de GC como repositórios digitais, portais, intranets, internets, plataformas de comunidade de prática virtuais e gestão eletrônica de documentos;

- Criar uma estrutura tecnológica voltada ao registro e a disseminação de informações e conhecimentos em todas as áreas da organização, inclusive as que se encontram distribuídas remotamente.

\section{Mensuração dos Resultados}

- Criar indicadores para verificar se o conhecimento está sendo utilizado para gerar benefícios;

- Verificar a aderência da gestão do conhecimento aos objetivos de negócio; 
- Divulgar os resultados alcançados pela gestão do conhecimento para que os indivíduos percebam os benefícios gerados por essa gestão;

- $\quad$ Desenvolver métricas para avaliar os investimentos em gestão do conhecimento;

- Mensurar os resultados alcançados pela GC sob as perspectivas financeira, operacional e estratégica;

- Criar indicadores para verificar se a memória organizacional está sendo utilizada para gerar benefícios;

- Criar indicadores para verificar se a memória organizacional está sendo alimentada e utilizada de maneira efetiva;

- $\quad$ Desenvolver métricas para avaliar os investimentos em memória organizacional;

- Mensurar os resultados alcançados pela MO sob as perspectivas financeira, operacional e estratégica.

\section{Alta administração}

- Reconhecer o conhecimento como 0 ativo organizacional mais importante;

- $\quad$ Fazer com que todos na organização compreendam o valor da gestão do conhecimento e da memória organizacional para a obtenção de vantagem competitiva;

- C Criar a M0 para preservar o conhecimento e retê-Io para seu uso posterior;

- Envolver-se em atividades que os levem a compreender sobre 0 escopo da gestão do conhecimento e da memória organizacional na organização;

- $\quad$ Fazer com que os dirigentes organizacionais se comprometam com a GC e com a MO;

- $\quad$ Fazer com que o conhecimento seja disseminado e 0 compartilhado em todos os níveis hierárquicos;

- Oferecer condições para que as mudanças necessárias se tornem efetivas e levem à sustentabilidade no longo prazo;

- $\quad$ Fazer com que os indivíduos compreendam a informação a partir de seu contexto exterior, compreendam o que é a organização e o que ela faz, ressaltando sua visão, missão, valores e cultura organizacional;

- C C C Ciar na organização e em todos que a compõe a visão de que o conhecimento é essencial para alcançar os objetivos organizacionais desejados; 
- Encorajar a participação entre os indivíduos, criando condições para que eles se sintam à vontade para participar caso tenham alguma ideia, sem medo de repreensões;

- Divulgar o conhecimento criado localmente para todos os interessados, inclusive entre organizações parceiras;

- Estimular a compreensão e o respeito pelo valor do conhecimento contribuído, por meio de políticas explícitas sobre a utilização dos ativos intelectuais;

- Apresentar os benefícios da GC e da MO a toda a organização;

- Identificar oportunidades estratégicas para a implantação da GC e da MO;

- Estabelecer um sistema de reconhecimento e de recompensas pela melhoria do desempenho, aprendizagem e criação de novos conhecimentos;

- Mobilizar os demais níveis hierárquicos, por meio do suporte ao compartilhamento do conhecimento, da criação de condições internas para a disseminação do conhecimento, definição clara de metas, objetivos e resultados esperados e da liberdade de interação entre as pessoas e grupos;

- Dar suporte necessário para a gestão do conhecimento, uma vez que sua implantação requer uma profunda alteração organizacional;

- $\quad$ Alocar recursos financeiros para viabilizar os projetos de gestão do conhecimento e garantir a utilização do conhecimento para a melhoria de processos, produtos e serviços;

- Definir a política de proteção do conhecimento;

- Ser exemplo de compartilhamento do conhecimento e de trabalho colaborativo;

- Abrir caminhos para obtenção de conhecimento em contexto externo à organização.

\section{Liderança}

- Reconhecer o conhecimento como 0 ativo organizacional mais importante;

- Desenvolver atividades que permitam capturar o conhecimento não documentado e integrá-lo à memória organizacional;

- Incentivar a delegação e a autonomia;

- $\quad$ Fazer com que os indivíduos compreendam o real valor da gestão do conhecimento e da memória organizacional para a obtenção de vantagem competitiva;

- $\quad$ Disseminar a visão de que o conhecimento é essencial para alcançar as metas traçadas; 
- Demonstrar que a GC é um processo voltado para o conhecimento individual e coletivo, que busca alcançar não apenas os objetivos organizacionais, mas também os desejos individuais de carreira e aprendizado;

- $\quad$ Encorajar a participação entre os indivíduos, criando condições para que eles se sintam à vontade para participar caso tenham alguma ideia, sem medo de repreensões;

- $\quad$ Apresentar e reforçar a visão, os objetivos e as estratégias da gestão do conhecimento;

- $\quad$ Ser exemplo de compartilhamento do conhecimento e de trabalho colaborativo;

- $\quad$ Estimular a criação e a manutenção da MO para reduzir a perda de conhecimento organizacional;

- $\quad$ Estimular os líderes a participarem de redes sociais para terem acesso ao conhecimento que a organização necessita;

- Desenvolver competências de liderança em seus colaboradores para que estes possam influenciar, de forma positiva, os processos de criação, compartilhamento, armazenamento e uso de conhecimento.

\section{Processos}

- $\quad$ Desenvolver políticas e normas para criação, compartilhamento, armazenamento e uso de conhecimento;

- Criar processos para identificar o conhecimento necessário para 0 desenvolvimento organizacional;

- $\quad$ Desenvolver processos que propiciem a criação do conhecimento organizacional;

- $\quad$ Desenvolver processos que estimulem e facilitem o compartilhamento do conhecimento;

- $\quad$ Criar processos que incentivem a aplicação do conhecimento;

- $\quad$ Criar processos que levem a reutilização do conhecimento;

- Desenvolver processos para a criação e manutenção da memória organizacional;

- $\quad$ Criar processos que propiciem a inclusão de lições aprendidas e melhores práticas como parte da memória da organização;

- Desenvolver processos que sistematizados e modelados com o conhecimento adequado contribuem para melhorar o desempenho organizacional.

\section{Estratégia}

- $\quad$ Garantir que as práticas de gestão do conhecimento sejam executadas e que estejam alinhadas com as demais práticas organizacionais; 
- Garantir que a criação e a manutenção da memória organizacional estejam alinhadas com as demais praticas organizacionais;

- Identificar informações internas e externas de que o organização necessita para o desenvolvimento de suas atividades;

- $\quad$ Fazer com que as informações que a organização necessita sejam armazenadas na memória organizacional de modo que estas possam ser utilizadas sempre que necessário;

- Conscientizar a organização de suas capacidades, deficiências e necessidades;

- Mediar as relações com clientes, fornecedores, concorrentes e parceiros que são importantes fontes de conhecimento para as organizações;

- Analisar se a distribuição do conhecimento dentro da organização faz com que o conhecimento do indivíduo seja transferido para a organização, além de garantir que o conhecimento esteja disponível para todos que dele necessitem;

- Desenvolver procedimentos para que as habilidades e ativos de conhecimentos sejam colocados em uso e a serviço da organização;

- $\quad$ Armazenar e atualizar o conhecimento por meio da constituição da memória da organização para que o conhecimento não seja perdido dentro da organização;

- Verificar se o conhecimento é útil, válido e se vai gerar valor para a organização;

- Incentivar as práticas de benchmarking, observação, entrevistas, educação corporativa, entre outras, para aumentar a base de conhecimento da organização;

- Definir as áreas de conhecimento nas quais os indivíduos devem focar o aprendizado;

- Manter a estratégia empresarial de modo claro;

- Definir metas que sejam desafiadoras e motivadoras com a finalidade de analisar a relação que se desenvolve entre conhecimento organizacional e estratégia corporativa;

- Alinhar a GC com a visão de futuro, a missão institucional, os objetivos estratégicos e as metas, de modo que possa servir como instrumento para alcançar os resultados organizacionais;

- Disponibilizar para a organização o fundamento de como suas capacidades e recursos devem ser desenvolvidos para que os objetivos da GC possam ser alcançados.

\section{Infraestrutura}

- Construir uma rede de interações fortemente baseada na confiança e na solicitude entre os indivíduos que compõem a organização; 
- $\quad$ Conceber um meio propicio para o intercâmbio de ideias, experiências, opiniões e crenças pessoais;

- $\quad$ Propiciar a criação e o desenvolvimento da memória organizacional;

- $\quad$ Criar um contexto que impulsione a criação do conhecimento e que possibilite o desenvolvimento de relacionamentos sólidos e de colaboração para que o compartilhamento do conhecimento ocorra de modo eficaz;

- $\quad$ Criar estruturas e processos que promovam a reutilização do conhecimento por meio da M0 para que a infraestrutura de conhecimento seja abrangente e bem organizada;

- $\quad$ Criar uma estrutura organizacional onde as comunicações, a MO e os fluxos de conhecimento possam ocorrer de modo eficiente e, assim, permitir que as ideias fluam tanto para dentro como para fora da organização, gerando diferentes perspectivas e possibilidades;

- Incorporar novas estruturas na gestão organizacional e na memória organizacional para avaliar a base de recursos de geração de valor;

- $\quad$ Proporcionar alto grau de autonomia às equipes de trabalho e valorizar as competências individuais;

- Desenvolver estruturas organizacionais que se constituem em um processo contínuo de adequação ao contexto de mercado;

- $\quad$ Criar estruturas que promovam a descentralização de tarefas, a autonomia e a delegação de poder para a motivação para 0 aprendizado, redução de níveis hierárquicos e desempenho vinculado à satisfação dos clientes.

\section{Recursos}

- $\quad$ Alocar tempo para que os indivíduos possam compartilhar conhecimento;

- $\quad$ Alocar tempo para que os indivíduos possam atualizar e utilizar a memória organizacional;

- Estabelecer um espaço, físico ou virtual, para que o compartilhamento do conhecimento aconteça;

- Alocar recursos para a obtenção de conhecimento por meio de compra ou desenvolvimento de conhecimento intra-organização, parcerias com universidades, contratação de consultorias e comunidades de práticas;

- Administrar os recursos de modo que reabasteçam e criam conhecimento organizacional por meio da memória organizacional; 
- Alocar recursos para a criação e manutenção da memória organizacional;

- Disponibilizar recursos financeiros necessários para a operacionalização da GC e da MO;

- Disponibilizar indivíduos adequados e preparados para o desempenho da gestão do conhecimento e da memória organizacional;

- Obter o compromisso da alta administração quanto à atribuição dos recursos necessários para a realização dos processos de gestão do conhecimento e da memória organizacional.

\section{CONSIDERAÇÕES FINAIS}

A partir da análise dos dados se infere que as empresas do setor cárnico de Salamanca não dispunham de atividades e processos formais voltados a gestão do conhecimento e a memória organizacional, bem como desconheciam a abrangência e a profundidade dos principais conceitos envolvidos com GC e MO. Apesar de reconhecerem a importância da GC voltada a MO e de disporem de ambientes propícios a implantação desta gestão, verificou-se que as ferramentas de TIC, fundamentais para a implantação e manutenção da GC com características da M0, não eram utilizadas conforme suas potencialidades.

Diante da importância e da relevância do conhecimento para as organizações, o principal resultado a ser destacado é o desenvolvimento de diretrizes estratégicas baseadas nos FCS da GC que considerem as características da MO, de modo a contribuir de forma eficaz para o sucesso da implantação e do desenvolvimento da GC nas organizações.

As principais contribuições deste estudo para a área acadêmica estão relacionadas com a realização de estudos aprofundados acerca da GC voltada à memória organizacional e de diretrizes estratégicas baseadas nos FCS da GC que considerem as características da MO. Com a proposição destas diretrizes, acredita-se que novas fronteiras no campo da GC possam ser pesquisadas e descobertas na área da Cl. Espera-se que a realização desta pesquisa contribua para realização de mais pesquisas nesta área na $\mathrm{Cl}$ que ainda é recente e que, portanto, carece de mais estudos.

Em relação à área gerencial, este estudo contribui com a identificação de aspectos essenciais para 0 sucesso da implantação e do desenvolvimento da GC por meio da proposição de diretrizes estratégicas baseadas nos FCS da GC que considerem as características da M0, de modo que as organizações possam usufruir dos benefícios desta gestão, além de construir uma vantagem competitiva sustentável em um contexto altamente competitivo. 


\section{AGRADECIMENTO}

À Fundação de Amparo à Pesquisa do Estado de São Paulo (FAPESP) pelo apoio financeiro essencial para a realização dessa pesquisa.

\section{REFERÊNCIAS}

ANGELONI, M. T. (Org.). Organizações do conhecimento: infra-estrutura, pessoas e tecnologia. 2. ed. São Paulo: Saraiva, 2008.

ARBONÍES, Á. Conocimiento para innovar. España: Díaz de Santos, 2006.

ARBONÍES, A.; ALDÁZABAL, J. Propuesta para el cálculo del índice de memoria de inteligencia organizacional (IMIO) basado en el MIK K-FACTS. Barcelona, España: Sussex University, 2005.

Asociación Nacional de Industrias de la Carne de España (ANICE). Disponível em: http://www.anice. es. Acesso em 13 mar. 2019.

BARDIN, L. Análise de conteúdo. Lisboa: Edições 70, 2011.

BATISTA, F. F. Modelo de gestão do conhecimento para a administração pública brasileira: como implementar a gestão do conhecimento para produzir resultados em benefício do cidadão. Brasília: Ipea, 2012.

BENAVIDES, C; QUINTANA C. Gestión del conocimiento y calidad total. Madrid, España: Díaz de Santos, 2003.

BENNET, A.; BENNET, D. The intelligent complex adaptive system. New York: Elsevier Butterworth Heinemann, 2004.

BESEN, F. Fatores críticos de sucesso na gestão do conhecimento. In: International conference on interactive computer aided blended learning, 2013, Florianópolis. Anais... Florianópolis: IBCL, 2013.

BUKOWITZ, W.R.; WILLIAMS, R.L. Manual de gestão do conhecimento: ferramentas e técnicas que criam valor para a empresa. Porto Alegre: Bookman, 2002. 399p.

CASTILLO, L. A. M.; CAZARINI, E. W. Modelo integrado para a implantação da gestão do conhecimento. GEPROS: Gestão da Produção, Operações e Sistemas, v.4, n.4, p.61-77, 2009. Disponível em: https:// revista.feb.unesp.br/index.php/gepros/article/viewFile/850/281. Acesso: 14 mai. 2019.

CHONG, S. C.; CHOI, Y. S. Critical factors in the successful implementation of knowledge management. Journal of Knowledge Management Practice, v. 6, 2005.

CORREA, F.; ZIVIANI, F.; MARTINS, H. C. A gestão do conhecimento holística: análise de aderência do modelo de teixeira filho (2000). Perspectivas em Gestão \& Conhecimento, v. 8, n. Especial, p. 167182, 2018. 
DALKIR, K. Knowledge management in theory and practice. Elsevier, USA: Linacre House, Jordan Hill, Oxford, 2005.

DAVENPORT, T. H., PRUSAK, L. Conhecimento empresarial. Rio de Janeiro: Campus, 1998.

DEL-MASSA, H. C. 0.; DAMIAN, I. P. M.; VALENTIM, M. L. P. Competência em informação no apoio à gestão do conhecimento. Informação \& Sociedade: Estudos, v. 28, n. 1, 2018.

EARL, M. Knowledge Management Strategies: Towards a Taxonomy. Journal of Management Information Systems, Summer, v. 18, n. 1, 2001.

FREIRE, P. de S. et al. Memória Organizacional e seu papel na Gestão do Conhecimento, Revista de Ciências da Administração, v. 14, n. 33, p. 41-51, ago 2012.

FRESNEDA, P. S. V. et al. Diagnóstico da gestão do conhecimento nas organizações públicas utilizando o método organizational knowledge assessment (OKA), II Congresso Consad de Gestão Pública - Painel 20: Gestão do conhecimento e inovação para a melhoria da gestão pública. 2009. Disponível em: << http://consad.org.br/wp-content/uploads/2013/02/DIAGN\%C3\%93STICO-DAGEST\%C3\%830-DO-CONHECIMENTO-NAS-ORGANIZA $\%$ C3 $\% 87 \%$ C3\%95ES-P\%C3\%9ABLICASUTILIZAND0-0-M\%C3\%89TOD0-ORGANIZATIONAL-KNOWLEDGE-ASSESSMENT-OKA2.pdf >>. Acesso em: 22 set. 2019.

FURLANETTO, A.; OLIVEIRA, M. Fatores estratégicos associados às práticas de gestão do conhecimento, Análise, Porto Alegre, v. 19, n. 1, p. 99-123, jan./jun. 2008.

GARCIA; M. A.; POSSAMAI, 0. Gestão do conhecimento no processo de desenvolvimento de produtos: modelo para a identificação dos fatores críticos de sucesso. In: ENCONTRO NACIONAL DE ENGENHARIA DE PRODUÇÃO, 2009, Bahia. Anais... Bahia: ENEP, 2009.

GNECCO JR., L. et al. Gestão do Conhecimento: fatores críticos de sucesso, REUNA, Belo Horizonte, v.15, n.1, p. 47-64, jan.-abr. 2010.

HOFFMAN, W. A. M. Gestão do conhecimento e da informação em organizações baseados em inteligência competitiva. Ciência da Informação, v. 45, n. 3, 2016.

HOLSAPPLE, C. W.; JOSHI, K. D. Description and analysis of existing knowledge management frameworks. In: Systems Sciences, 1999. HICSS-32. // Proceedings of the 32nd Annual Hawaii International Conference on. IEEE. https://doi.org/ 10.1109/HICSS.1999.772796

HUNG, C. Y.; HUANG, M. S.; LIN, P. Q.; TSAI, L. M. Critical factors in adopting a knowledge management system for the pharmaceutical industry. Industrial Management \& Data Systems, v. 105, n. 2, p. 164-183, 2005.

ISO International Standardization Organization. ISSO 30301: 2019. Information and Documentation. Management System for Records.Requirements. Géneve: ISO, 2016.

JUNTA DE CASTILLA Y LEÓN (JCyL). Estudio del Sector Cárnico en Castilla Y León. Disponível em: <http://www.castillayleon.cco0.es/7f9e3b8194b39f8a841a3628fee032f8000054.pdf>. Acesso: 13 mar. 2019. 
MELGAR-SASIETA, H. A. M.; BEPPLER, F. D.; PACHECO, R. C. S. A memória organizacional no contexto da engenharia do conhecimento, DataGramaZero, Rio de Janeiro, v. 12, n. 3, ago. 2011.

NASCIMENTO, N. M. do et al. Gerenciamento dos Fluxos de Informação como requisito para a preservação da Memória Organizacional: um diferencial competitivo, Perspectivas em Gestão \& Conhecimento, v. 6, Número Especial, p. 29-44, jan. 2016.

NASCIMENTO, N. M.; VITORIANO, M. C. C. P. 0 estudo da produção documental e a memória organizacional em ambientes empresariais, Em Questão, v. 23, n. 1, p. 202-227, 2017.

NEVES, P. M. C.; CERDEIRA, J. P. Memória organizacional, gestão do conhecimento e comportamentos de cidadania organizacional. Perspectivas em Gestão \& Conhecimento, v. 8, n. 1, p. 3-19, 2018.

NONAKA, I.; TAKEUCHI, H. Criação de conhecimento na empresa: como as empresas japonesas geram a dinâmica da inovação. 29.ed. Rio de Janeiro: Elsevier, 1997.

PEREIRA, M. O. F.; SILVA, H. de F. N.; PINTO, J. S. de P. A Memória Organizacional nos processos de Gestão do Conhecimento: um estudo na Universidade Federal do Paraná, Informação \& Informação, Londrina, v. 21, n. 1, p. 348 - 374, jan./abr. 2016.

PÉREZ-MONTORO GUTIÉRREZ, M. Gestión del conocimiento en las organizaciones: fundamentos, metodología y praxis. Gijón; Trea, 260p., 2008.

RIVERA, G.; RIVERA, I. Design, Measurement And Analysis Of A Knowledge Management Model In The Context Of A Mexican University. Innovar, Bogotá, v. 26, n. 59, p. 21-34, Jan. 2016.

SANTOS, J. C. dos; MORO-CABERO, M. M.; VALENTIM, M. L. P. A Memória Organizacional como diferencial competitivo em ambientes organizacionais. Anais do XI Seminário de Pesquisa em Ciências Humanas, Londrina, 2016.

SOUZA, E. D. de; DIAS, E. J. W.; NASSIF, M. E. A gestão da informação e do conhecimento na Ciência da Informação: perspectivas teóricas e práticas organizacionais. Informação \& Sociedade: Estudos, João Pessoa, v.21, n.1, p.55-70, jan./abr. 2011. Disponível em: <http://www.periodicos.ufpb.br/ojs/ index.php/ies/article/view/4039/5598>. Acesso: 18 set. 2019.

SPILLER; A.; PONTES, C. C. C. Memória organizacional e reutilização do conhecimento técnico em uma empresa do setor eletroeletrônico no Brasil. RBGN - Revista Brasileira de Gestão de Negócios, São Paulo, v.9, n.25, p.96-108, set./dez. 2007.

TERRA, J. C. C. Gestão do conhecimento: o grande desafio empresarial. São Paulo: Negócio Editora, 2005.

WONG, Y. K. Critical success factors for implementing knowledge management in small and medium enterprises, Industrial Management \& Data Systems, v. 105, n. 3, p. 261-279, 2005. Disponível em: https://doi.org/10.1108/02635570510590101. Acesso: 22 ago. 2019.

WONG, K. Y.; ASPINWAAL, E. Knowledge Management Implementation Frameworks: A Review, Knowledge and Process Management, v. 11, n. 2, pp 93-104, 2004. 